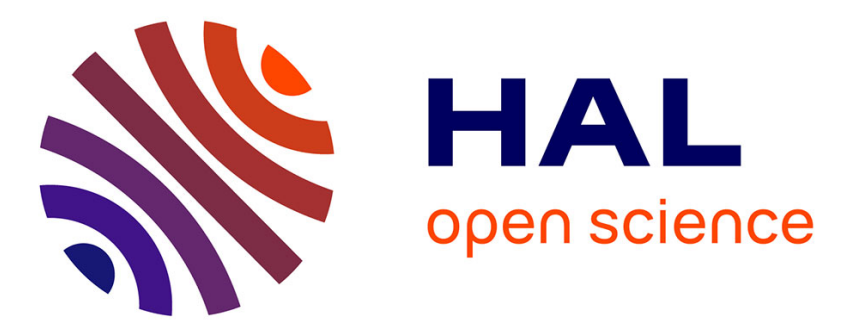

\title{
Pour une géographie des nostalgies citadines. Quelques pistes théoriques à partir d'exemples chiliens et sud-africains
}

\author{
Colin Clement, Philippe Gervais-Lambony
}

\section{To cite this version:}

Colin Clement, Philippe Gervais-Lambony. Pour une géographie des nostalgies citadines. Quelques pistes théoriques à partir d'exemples chiliens et sud-africains. Annales de géographie, 2019, 728, pp.37-56. 10.3917/ag.728.0037 . hal-02319699

\section{HAL Id: hal-02319699 \\ https://hal.parisnanterre.fr/hal-02319699}

Submitted on 18 Oct 2019

HAL is a multi-disciplinary open access archive for the deposit and dissemination of scientific research documents, whether they are published or not. The documents may come from teaching and research institutions in France or abroad, or from public or private research centers.
L'archive ouverte pluridisciplinaire HAL, est destinée au dépôt et à la diffusion de documents scientifiques de niveau recherche, publiés ou non, émanant des établissements d'enseignement et de recherche français ou étrangers, des laboratoires publics ou privés. 


\title{
Pour une géographie des nostalgies citadines. Quelques pistes théoriques à partir d'exemples chiliens et sud-africains
}

\author{
For a geography of urban nostalgia. Some theoretical \\ issues from Chilean and South African examples
}

\author{
Clément Colin \\ Professeur associé, Escuela de Trabajo Social/École de Travail Social, Pontificia Universidad \\ Católica de Valparaíso
}

Philippe Gervais-Lambony

UMR LAVUE, Université Paris Nanterre et Institut Universitaire de France

Résumé L'article vise à montrer comment la nostalgie peut être pensée comme une catégorie géographique d'analyse qui apporte de nouveaux éléments de compréhension de la ville, en particulier du monde urbain actuel caractérisé par la rapidité des processus de destruction créatrice. La notion, peu abordée jusqu'à récemment en géographie francophone, est porteuse de possibilités nouvelles en relation avec une géographie des émotions aujourd'hui en développement et plus largement avec les études mémorielles. Le texte s'appuie sur des exemples de terrains en Afrique du Sud et au Chili, à l'échelle du quartier, pour appuyer une proposition théorique. La réflexion s'organise en quatre parties : définitions de la nostalgie et de ses dimensions spatiales; relation entre le sentiment individuel et les discours portés collectivement ; lien entre nostalgie et temporalités du changement socio-spatial; mobilisation de la recherche sur les nostalgies comme outil de compréhension des situations de conflits liées à des représentations diverses du passé, du présent et du futur.

Abstract The article aims to show how nostalgia can be considered as a geographic category of analysis that brings new elements for understanding the city, especially in today's urban world characterized by rapid processes of creative destruction. The notion, rarely used in geography, brings new possibilities in relation to a geography of emotions currently in development and, more widely, to memorial studies. The paper is based on case studies in South Africa and Chile, at the neighbourhood level, to support a theoretical proposal. The reflection is organized in four parts : definitions of nostalgia and its spatial dimensions; interrelations between individual feeling and collective discourses; links between nostalgia and temporalities of socio-spatial change; mobilization of research on nostalgia as a tool for understanding conflict situations linked to various representations of past, present and future.

Mots-clés ville, nostalgie, identités, Chili, Afrique du Sud.

Keywords city, nostalgia, identities, Chile, South Africa. 
"Sous le pont Mirabean coule la Seine

Et nos amours

Faut-il qu'il m'en souvienne

La joie penait toujours après la peine »

Guillaume APOLLINAIRE, "Le Pont Mirabeau»

\section{Introduction $^{1}$}

Nous n'avons pas à notre disposition une définition univoque de la nostalgie. C'est une émotion ambivalente qui peut tout aussi bien renvoyer à des désirs de retours basés sur des souvenirs vécus et/ou partagés qu'à une projection vers un futur possible fondée sur une critique du présent au regard de passés évanouis (Boym, 2001). En revanche nous savons que foisonnent dans le monde contemporain les références au passé : "The past has become part of the present in ways simply unimaginable in earlier centuries [...] temporal boundaries have weakened just as the experiential dimension of space has shrunk» (Huyssen, 2003, p. 1). Une relation particulière au passé semble donc marquer notre époque, d'où les occurrences si fréquentes d'expressions de nostalgies, nostalgies de notre temps puisque comme toute émotion celle-ci a une histoire (Corbin et al., 2016). Nous savons aussi que cette présence du passé s'ancre dans la matérialité spatiale et est potentiellement productrice d'espaces (Blunt, 2003 ; Bonnett et Alexander, 2013 ; Wheeler, 2016). Comme l'avancent Kitson et McHugh (2015), la nostalgie est bien une émotion qui lie les individus à leur environnement matériel. Elle s'incarne dans des pratiques individuelles et collectives, engendrant une "esthétique du passé ", une forme spécifique de sentir et de percevoir liée à une relation à un passé dans le présent. Â ce titre, il nous semble qu'elle est bien un sujet pour la géographie et nous proposons de pointer quelques pistes pour une géographie des nostalgies citadines.

La raison première en est que nous avons rencontré des discours et des pratiques nostalgiques sur nos terrains de recherche respectifs et que, dès lors, la nostalgie nous est apparue comme pouvant être pensée comme une catégorie géographique d'analyse qui apporte de nouveaux éléments de compréhension de la ville. Non pas que les citadins auprès desquels nous avons conduit des enquêtes, par entretien ou par observation, emploient eux-mêmes le mot nostalgie, mais parce que leur propos sur le présent, les sentiments dont ils font état ou que nous observons à travers l'étude de leurs pratiques, se réfèrent à des temps et des lieux passés que, le plus souvent, ils valorisent. Pour illustrer notre propos, nous nous fonderons donc sur nos travaux de recherche réalisés, à l'échelle de quartiers urbains, pour l'un d'entre nous au Chili depuis 2013 (à Santiago et à Valparaiso) et pour l'autre en Afrique du Sud (à Johannesburg et à Ekurhuleni)

1 Les auteurs remercient CONICYT pour son appui à travers le projet « Redes en etapa incial convocatoria 2017 » REDI170126 et le projet FONDECYT Iniciación nº 11180372. 
depuis les années 2000. La démarche comparatiste, ne repose donc pas ici sur un choix raisonné des terrains, mais sur la coïncidence d'une rencontre ; cette coïncidence cependant, à n'en pas douter, s'inscrit dans un mouvement de la recherche en sciences sociales où les études mémorielles ont pris récemment une place majeure. La notion de nostalgie a été travaillée par la géographie culturelle et les études culturelles dans le monde anglophone (Bonnett, 2015), notamment en anthropologie (Angé et Berliner, 2015). Ces approches s'inscrivent dans l'ensemble plus vaste des travaux de sciences sociales sur les émotions. Ceux-ci se déploient en France depuis les années 1980 en anthropologie (Crapanzano, 1994) mais depuis peu la géographie des émotions a aussi gagné droit de cité (Guinard et Tratnjek, 2016). Mais faire le choix en géographie d'une telle entrée théorique et méthodologique c'est aussi se positionner dans un rapport au champ des études mémorielles (Lavabre, 2012). Champ d'ailleurs de plus en plus vaste au fur et à mesure que la diversité, voire la fragmentation, des mémoires se déploie (Lowenthal, 2015) et que les références au passé se multiplient tant dans le domaine classique du patrimoine que dans celui de la promotion touristique ou de la publicité et du marketing en général au point que l'on a pu qualifier notre époque post-moderne de «temps des imaginaires » où «la question n'est plus celle de la nostalgie, mais bien celle d'un imaginaire social largement construit sur des reprises » (Appadurai, 1996, p. 67).

Dans ce qui suit nous proposons de cibler notre propos sur la nostalgie sentie et exprimée par les habitants d'un territoire. Cela signifie aborder "par le bas » les interrelations complexes entre temporalités, spatialités et identités. Dans le contexte contemporain caractérisé par une tension permanente, au moins au niveau discursif, entre circulation et ancrage, mobilité et immobilité, travailler sur les nostalgies prend tout son sens. Nous proposons de mettre en évidence la richesse et la diversité des nostalgies comme facteur explicatif de nouvelles tensions dans le monde urbain. La nostalgie sera donc ici entendue comme forme sociale, au sens simmelien du terme, mais aussi comme relation affective au temps et à l'espace. Base de constructions identitaires multiples en lien avec des expériences de l'espace divergentes, son étude permet une meilleure compréhension des comportements et discours individuels et collectifs citadins.

Nous organisons ce qui suit en quatre parties où dialoguent approches théoriques et exemples de terrain. Tandis que la première partie porte sur les définitions de la nostalgie et ses liens avec la question spatiale, la seconde interroge la relation entre l'individuel et le collectif. Ensuite nous proposons de comprendre la nostalgie comme un phénomène intrinsèquement lié à la question des temporalités du changement socio-spatial, en particulier dans le monde urbain actuel caractérisé par la rapidité des processus de destruction créatrice, et comme outil pour comprendre des situations de conflits liées à des représentations diverses du passé, du présent et du futur. Enfin, nous proposons de conclure sur une ouverture plus générale en termes de géographie de l'expérience, la recherche sur les nostalgies ouvrant la voie, à notre avis, à une compréhension plus fine de la complexité de la relation humaine à l'espace et aux lieux. 


\section{Quelles typologies pour dire la diversité des nostalgies?}

$\mathrm{Si}$, entre nostos (retour) et algos (souffrance), la nostalgie est d'abord historiquement définie, en 1759 , dans le cadre du vocabulaire médical, pour désigner une maladie causée par l'exil, elle est le plus souvent présentée dans la littérature scientifique récente comme une réponse à la post-modernité caractérisée par une mobilité généralisée et une compression de l'espace-temps (Harvey, 1990 ; Massey, 1995). Elle a été aussi liée aux idées de retour et de conservatisme. Ainsi Tuan la dit caractéristique des sociétés inquiètes du changement, ce qui pourrait assez bien décrire notre présent : "When a people perceive that changes are occurring too rapidly, spinning out of control, nostalgia for an idyllic past waxes strong» (Tuan, 1977, p. 195). Elle est jugée dangereuse par certains auteurs qui n'y voient qu'une forme d'idéalisation du passé (Lewis, 1985), une falsification de l'histoire (Huyssen, 2003 ; Todorov, 2004). Mais la nostalgie n'est pas uniquement en lien avec le passé : elle est " activée » dans le présent et est donc liée aux imaginaires, représentations et idées partagées dans ce même présent (Pickering et Keighty, 2006). Il ne s'agit pas seulement d'un désir de conservation ou de restauration. Elle peut prendre différentes formes mais est toujours liée à une réinvention, réinterprétation ou reconstruction de passés dans le moment et le lieu présent. C'est pour cette raison même que l'on peut affirmer que la nostalgie n'est pas uniquement liée au temps mais aussi à l'espace et aux affects provoqués par les relations subjectives que l'individu, le groupe ou la société établit avec son environnement. On sait toute l'importance qu'accorde Maurice Halbwachs aux liens avec l'espace comme conditions même de la mémoire : "C'est l'image seule de l'espace qui, en raison de sa stabilité, nous donne l'illusion [...] de retrouver le passé dans le présent » (Halbwachs, 1950, p. 201). Ce qui revient à dire que la condition de la nostalgie est l'espace autant que le temps et que l'émotion nostalgique est une émotion spatio-temporelle.

Ce constat ne retire rien à la diversité des nostalgies selon les situations, les contextes et les échelles qui conduisent à des pratiques et discours divers selon les individus et les groupes sociaux. La nostalgie du migrant n'est par exemple pas la même que celle de la personne ancrée dans un unique lieu qui connaît de profonds changements. Ce constat est partagé par de nombreux auteurs qui, par conséquent, ont proposé des typologies des nostalgies. Même si chacune de ces typologies a ses limites, elles aident à mieux appréhender et questionner ce phénomène.

Boym (2001) a été une des premières auteures à s'intéresser à la diversité des nostalgies et à leur traduction en termes de pratiques. Elle fait une distinction entre ce qui serait une nostalgie restauratrice et ce qui pourrait être une nostalgie réflexive. La première serait liée à l'idée de retour à un «âge d'or », fondée sur les anxiétés, incertitudes et peurs contemporaines provoquées par un sentiment de perte d'identité. Elle servirait ainsi de base émotionnelle pour la reconstruction d'une «maison » perdue et se traduirait par exemple en discours et politiques nationalistes et parfois autoritaires fondés sur les mythes historiques fondateurs 
et une certaine idée des traditions nationales ou locales. Au contraire, la nostalgie réflexive s'appuie sur le passé pour penser le futur et se projeter dans l'avenir. Le nostalgique réflexif est amoureux de la distance qui le sépare d'un temps ou d'un espace perdu et il prend plaisir à la présence du passé dans le présent. Sa nostalgie n'est pas rejet du présent, elle est une refondation de soi à partir d'un regard critique et réflexif, et surtout d'une conscience du caractère éphémère du présent qui ouvre sur la possibilité de construire le futur. Ce double visage de la nostalgie se retrouve chez Barbara Cassin (2013) qui elle-même se réfère à la philosophie allemande pour distinguer une "nostalgie fermée ", celle de l'éternel retour, d'une "nostalgie ouverte ", qui ne revient pas en arrière mais qui «poursuit un objet indéterminé ou un idéal introuvable » (p. 60).

Suivant la même préoccupation, à partir de son travail de terrain en Afrique du Sud, l'un des deux auteurs du présent article (Gervais-Lambony, 2003) a proposé de distinguer trois types de nostalgies. Un premier serait prémoderne : il s'agirait de la nostalgie des exilés. Elle trouve sa source dans le déplacement, l'arrachement au lieu, c'est-à-dire le déracinement. Cette nostalgie du migrant est aussi, dans le cas urbain, le regret des lieux qui ont été détruits ou dont on a été expulsé. Un second type serait moderne : la nostalgie des citadins face au changement de leur environnement, en particulier face aux processus de destruction créatrice de la ville capitaliste (Harvey, 1990). Cette nostalgie, qui imprègne la poésie baudelairienne, se déploie surtout à partir du XIX ${ }^{\mathrm{e}}$ siècle du fait des changements provoqués par les révolutions industrielles en Europe, contexte historique dans lequel le mouvement romantique cultive sa fascination pour la ruine, l'antique et l'authentique. Finalement, la nostalgie post-moderne est le troisième type, c'est celle que l'on éprouve pour un passé ou un espace que l'on n'a jamais connu. Fondée sur les idées d'Appadurai (1996), cette dernière forme est la nostalgie pour un passé (ré) inventé et instrumentalisé par le marketing ou le politique.

S'intéressant à la patrimonialisation du centre de Luang Prabang (Laos), Berliner $(2012 ; 2013)$ propose, de son côté, une typologie qui distingue quatre catégories. Pour lui, tandis que les « bureaucrates » de l'UNESCO construisent une nostalgie institutionnalisée comme une valeur universelle à défendre à échelle globale à travers leurs discours hégémoniques sur la protection patrimoniale, les experts de terrain expriment, quant à eux, une "exonostalgie » qui se fonde sur un attachement cognitif et émotionnel fort aux lieux : ils font de la nostalgie un acte de militantisme. De leur côté, les habitants, selon leur trajectoire de vie et leur milieu socio-culturel, expérimentent des «endonostalgies» différenciées vis-à-vis du centre historique de la ville. Enfin, les touristes associent au site de Luang Prabang une nostalgie basée sur un pouvoir d'imagination et une quête d'authenticité instrumentalisée par les acteurs du marché économique du tourisme.

Ces typologies ne doivent cependant nous faire oublier ni les recouvrements entre catégories, ni les nuances internes à chaque catégorie, ni le caractère dynamique des processus nostalgiques. Le sentiment nostalgique est en effet mobile et entrecroisé. Les nostalgies restauratrice et réflexive de Boym peuvent par 
exemple coexister et les pratiques institutionnelles de protection ou conservation peuvent se maintenir dans une relation complexe et durable avec une perception plus personnelle et intime de la valeur donnée au passé. À partir d'entretiens menés auprès d'ex-résidents de Tyneside (proche de New Castle, UK) qui vivent depuis désormais 10 ans dans la vallée de Tyne (à environ $35 \mathrm{~km}$ de Tyneside), Bonnett et Alexander (2013) questionnent directement les relations entre passé, présent et futur dans les représentations et usages actuels de la ville. Tandis que les politiques de préservation patrimoniale participent à la perpétuation du lien affectif que les habitants éprouvent pour la ville d'avant dans la période actuelle, la modernisation et le développement de cette ville maintiennent une activité économique qui attire ces ex-résidents, perpétuant ainsi leur lien d'usage avec celle-ci. Dans ce cadre, la nostalgie prend forme dans les usages actuels : tout en allant "consommer ", les citadins retournent dans les lieux auxquels ils sont attachés. Cette étude montre ainsi la manière dont ces différentes formes de nostalgie se complètent et s'entrecroisent dans les discours et les pratiques des interviewés. Tandis que les politiques de préservation (nostalgie restauratrice) favorisent le maintien des souvenirs de «comme c'était avant ", les nouveaux usages que les ex-habitants font de la ville favorisent la perpétuation de leur lien affectif avec celle-ci (nostalgie réflexive). Sans ce nouvel usage économique, ce lien se romprait. À l'inverse, sans la protection patrimoniale, le «comme c'était avant » serait plus difficile à se représenter et se réimaginer.

Les différents efforts de catégorisation des nostalgies mettent finalement en évidence aussi les interrelations entre catégories. Les nostalgies sont complexes, multiples et dynamiques, elles font entrer en dialogue le spatial et le temporel, le politique et le social, l'historique et le mémoriel, le réel et l'imaginaire. Un dialogue qui n'est pas exempt de conflit ni de tension et qui révèle la complexité des relations affectives aux lieux et aux temps des sociétés humaines, des groupes sociaux et des individus. Un dialogue aussi dans lequel les relations entre l'individuel et le collectif font question : comment passe-t-on de l'un à l'autre?

\section{Nostalgies individuelles et constructions identitaires collectives}

La nostalgie, puisque liée à la mémoire, concerne l'individuel comme le collectif. Maurice Halbwachs (1950) propose de distinguer d'abord les effets différents des nostalgies dans un passage de La Mémoire collective (sans pour autant employer le mot nostalgie). Il distingue l'émotion de l'habitant "pour qui ces vieux murs, ces maisons décrépies, ces passages obscurs et ces impasses faisaient partie de son petit univers, et dont bien des souvenirs se rattachent à ces images maintenant à jamais effacées, sent que toute une partie de lui-même est morte avec ces choses » de celle du groupe qui «ne se contente pas de manifester qu'il souffre [...] il résiste de toute la force de ses traditions [...] il essaie de se maintenir ou de se 
reformer dans un quartier ou dans une rue qui ne sont plus faits pour lui, mais sur l'emplacement qui était le sien » (p. 201).

À l'échelle individuelle, la nostalgie serait donc activée par les matérialités rencontrées dans le quotidien. C'est en effet par la vue d'objets, de sites, d'édifices (et tout particulièrement dans des phases de transformation de l'espace) que l'habitant peut vivre une réminiscence ou plus exactement une reviviscence, pour reprendre le terme de Georges Poulet (1963) pour décrire cette expérience proustienne : l'émergence soudaine du passé dans le présent. Il s'agit, pour reprendre les mots de Proust, d'une "mémoire involontaire » engendrée par les perceptions du corps à partir desquelles s'effectue une analogie entre ce qui est perçu maintenant et ce qui le fut dans le passé, et qui donc fait " revivre » une expérience passée. Ces restes concrets de temps passés, présents dans l'espace actuel, sont ce que De Certeau (1980) nomme les « revenants de la ville ». Il peut s'agir d'un objet ou d'un édifice aujourd'hui inutile, telle une cabine téléphonique, une ancienne station de bus ou une simple inscription sur un mur. Il peut aussi s'agir de lieux abandonnés, un ancien bâtiment industriel qui rappelle un temps glorieux de l'économie locale ou nationale, une ruine, une friche. Selon De Certeau, ces restes poétisent le monde et ainsi le rendent habitable parce que non entièrement compréhensible (il les oppose aux monuments patrimonialisés). C'est dans cette droite ligne qu'a pu être développée une réflexion sur les tomasons (Gervais-Lambony, 2017) : on doit cette notion à Genpei Akassegawa (1987), artiste japonais d'hyperart. Pour désigner la catégorie d'objets urbains qu'il veut dévoiler il a repris le nom de Gary Thomasson, joueur de baseball états-unien « acheté » par une équipe japonaise. Une fois au Japon, Gary Thomasson s'est avéré inutile, incapable de jouer correctement, ombre de lui-même... Les tomasons d'Akassegawa sont des objets urbains dont la fonction n'est plus connue mais qui s'attardent dans nos espaces, escalier ne débouchant sur rien, panneau indiquant un lieu qui n'existe plus, tuyau sans usage dépassant d'un mur, etc. Mais on peut changer d'échelle et au-delà des objets considérer comme tomason des espaces, des quartiers... Le tomason est alors un concept utile en géographie parce qu'il permet de désigner ces objets et espaces urbains qui ne sont " pas à leur place ", dont on sait qu'ils sont des restes d'un passé presqu'oublié qui provoquent une nostalgie réflexive (et «involontaire » pour reprendre la distinction proustienne). En effet, le tomason ne peut faire songer à un retour en arrière, il est bien un signe du caractère éphémère du présent car on sait aussi cet objet lui-même appelé à disparaître.

Cette approche de la nostalgie comme émotion provoquée par une expérience spatiale est complémentaire d'une autre qui concernerait une nostalgie portée par la «mémoire volontaire » : la nostalgie est alors provoquée par un regard rétrospectif sur le passé, sur les lieux où l'on a vécu ou sur le lieu dans lequel on vit comparé au souvenir que l'on en a. Cette forme de nostalgie est plus restauratrice que réflexive car elle conduit à la mise en œuvre d'une résistance au changement telle que décrite par Halbwachs. N'avons-nous pas ici la meilleure piste pour comprendre les modalités du passage d'un sentiment individuel à une 
construction sociale collective liée au changement socio-spatial ? Les nostalgies individuelles sont sans doute nécessaires pour que puissent se construire un discours collectif sur le passé et des pratiques collectives de remémoration qui supposent la (ré) activation de « revenants ». Mais ce discours et ces pratiques collectives participent à la reconstruction du passé sur la base de la «mémoire volontaire ".

Ainsi se comprend mieux que ces formes collectives de nostalgie volontaire jouent un rôle important dans les pratiques de résistance culturelle ou de recherche de reconnaissance de l'identité d'un groupe (leur caractère alors de construction politique, d'invention ou de manipulation ne retirant d'ailleurs rien à la réalité des émotions ressenties). Dans ce cadre, la nostalgie est un mode d'affiliation sociale et de construction identitaire choisie (Berdahl, 1999; Bissell, 2005 ; Berliner, 2012 ; Colin, 2016). Il s'agit ainsi d'une nostalgie de résistance. Par leur revalorisation matérielle, les traces du passé devenues marques (Veschambre, 2008), passent de l'affectif au politique.

\section{Le Cas D'un Quartier de Santiago Centre}

Le centre de Santiago est depuis les années 1990 en proie à une pression immobilière sans précédent. Résultat d'une politique pro-entreprenariale où les pouvoirs publics (municipalité et État) jouent un rôle de facilitateurs du marché immobilier à travers la planification de la rénovation urbaine, les quartiers résidentiels composés de maisons d'un ou deux étages en façades continues se transforment en des zones constituées de tours dépassant parfois les quarante étages. II s'agit pour les habitants établis depuis plusieurs générations à la fois d'un changement matériel et d'une transformation du paysage social, des milliers de nouveaux résidents vivant désormais dans ces espaces autrefois peu denses. Face à cette situation, des protestations d'habitants se sont multipliées. Afin d'étudier ces groupes, un travail de terrain a été conduit (Colin, 2016) dans un quartier central de Santiago encore relativement épargné par les projets immobiliers et où la mobilisation collective a conduit à la déclaration d'une partie du quartier comme zone patrimoniale protégée en décembre 2015. C'est lors de cette enquête que la nostalgie s'est imposée progressivement comme thématique centrale pour comprendre ces mobilisations. Elle s'est révélée aussi bien dans les discours des défenseurs du quartier centrés sur " c'était mieux avant " que dans leurs comportements et réactions durant les réunions organisées par les groupes mobilisés. C'est dans ce contexte que la nostalgie apparaît à la fois comme un élément structurant de la mobilisation d'habitants et comme producteur de nouveaux espaces urbains.

Dans ce cadre, une double dynamique a conduit à la production de ce quartier comme un «espace nostalgique». La première est liée à l'expérience de l'espace. Elle se fonde sur les émotions provoquées par la réminiscence qu'expérimentent les habitants face aux changements socio-territoriaux. La nostalgie se matérialise alors de manière éphémère à travers des appropriations ponctuelles mais quotidiennes de l'espace qui peut aller de l'occupation le soir venu de l'espace public pour converser avec les voisins à des expositions photographiques anciennes et patrimoniales du quartier sur la place principale ou dans des centres culturels du secteur. Une seconde dynamique est institutionnelle. Il s'agit de protéger juridiquement le quartier par sa patrimonialisation. Elle se base sur une remémoration de passés effectuée à partir d'une construction collective qui se matérialise de manière permanente et marque l'espace, non seulement 
par l'application de normes et de règles de constructions ou de maintiens des édifices mais aussi par une revalorisation matérielle conduite par les habitants eux-mêmes.

À partir de l'étude de cette double dynamique à Santiago, il est possible d'avancer que la territorialisation de la nostalgie se fonde sur une appropriation matérielle et idéelle du quartier qui intègre les participants à la mobilisation mais exclut les personnes qui ne partagent pas cette nostalgie pour un temps passé. La revalorisation d'un «comme c'était avant» est ici intimement liée à une recherche identitaire qui conduit à la co-construction de normes sociales et de règles comportementales entre habitants défendant le quartier face à la pression immobilière et à l'arrivée de nouveaux venus dits « étrangers ». L'espace de la nostalgie serait donc le résultat d'une double opposition entre, d'un côté, les espaces vécus des habitants et les espaces conçus par les institutions (ici la protection juridique par le Conseil des Monuments Nationaux et la planification urbaine de la Municipalité) et d'un autre côté, entre les imaginaires différents des anciens et des nouveaux résidents. Les habitants « anciens » du quartier expriment leurs nostalgies individuelles pour un passé suffisamment proche pour qu'ils s'en souviennent. Ces nostalgies rencontrent un écho collectif au moment même où ces espaces sont en danger puisque mis en question par des processus sociaux et/ou économiques. En devenant collectives, ces nostalgies se transforment et prennent un autre sens pour les habitants affectés, elles servent de base à la revendication d'un «droit de mémoire » considéré comme faisant partie du «droit à la ville » (Didier, 2018). Il ne s'agit plus seulement de l'expression d'un regret mais bel et bien de la co-construction d'une revendication sociale : un droit à conserver ces espaces comme ils étaient. Cependant, ces revendications révèlent une brèche entre l'ancien habitant établi qui a connu l'avant de référence et le résident plus récent qui représente les nouveaux usages possibles de ces espaces et qui incarne donc le danger de changement. C'est dans ce contexte que l'étude des nostalgies conduit le chercheur à questionner les limites entre l'habitant légitime et l'illégitime, c'est pourquoi aussi le sujet de la nostalgie est lié directement à des questions de droits et de justice spatiale.

\section{Nostalgie et changement socio-spatial}

La nostalgie est souvent associée au déplacement géographique et plus exactement à l'exil, c'est-à-dire à l'arrachement à un lieu, au déracinement. Cependant, comme on vient de le voir, elle est aussi le fait de citadins qui n'ont pas vécu de déplacement. Mais dans les deux cas c'est le changement qui provoque la nostalgie. En effet, si les citadins venus d'une autre ville, d'une zone rurale, d'un autre pays, ou simplement d'une autre partie de la même ville, se sont déplacés dans l'espace, ceux qui sont restés dans le même lieu se sont déplacés dans le temps, et leur espace de vie a changé. Le regard porté sur le passé peut-être donc être nostalgique soit du passé, soit du lointain. L'expérience humaine commune 
est ici celle du "déplacement », au sens de changement de lieu ou de changement du lieu où l'on est resté (Gervais-Lambony et Desbois, 2017). Cette expérience du déplacement est un des caractères même de l'expérience citadine qui est un constant processus d'adaptation au changement. Et ce, bien plus vigoureusement aujourd'hui que jadis dans la mesure où la ville contemporaine est à la fois plus multiculturelle (avec des flux de personnes accrus) et connait des processus de transformation spatiale plus rapides. Dès lors l'émotion nostalgique est bien plus partagée qu'il ne semble par les «mobiles» et les «immobiles» : en un même lieu se rencontrent la nostalgie pré-moderne de l'exilé et la nostalgie moderne née de la transformation de l'espace (Gervais-Lambony, 2003). Ceci se traduit par une grande complexité des nostalgies à l'échelle locale dans la mesure où coexistent des groupes différents dans le même espace et où « il y a autant de façons de se représenter l'espace qu'il y a de groupes » (Halbwachs, 1950, p. 232). Nous l'exemplifierons à partir de deux cas : un quartier résidentiel de Valparaíso, étudié par l'un des auteurs, et celui de la nostalgie des nouvelles classes moyennes sud-africaines ayant quitté le township où étaient cantonnés les citadins «non-blancs » sous l'apartheid pour la suburb naguère réservée aux populations «blanches » abordé à travers l'étude de textes littéraires sud-africains.

\section{NOSTALGIE ET GÉNÉRATIONS DANS UN QUARTIER DE VALPARAíSO}

Le quartier Esperanza est un cerro (une colline) situé en face de l'Océan Pacifique, dans la ville de Valparaíso. Port principal du Chili, cette ville se structure en deux parties géographiques distinctes: tandis que le plano (le plan) concentre la grande partie des activités économiques, les habitants, appelés les porteños, vivent dans les quarante-quatre cerros (collines) qui l'entourent. Valparaíso a connu un grand développement économique durant le $\mathrm{XIX}^{\mathrm{e}}$ siècle grâce à l'arrivée de familles commerçantes européennes travaillant en lien avec le port. Le tremblement de terre de 1906 qui a détruit $85 \%$ de la ville, l'inauguration du canal de Panama en 1914 qui change les routes maritimes commerciales et la crise des années 1930 ont ensuite inversé la dynamique, produisant chômage et pauvreté et conduisant à une nostalgie collective de la gloire passée. Durant la dictature de Pinochet (1973-1990), les politiques urbaines libérales ont dérégularisé le marché immobilier, provoquant une forte pression immobilière, qui s'est renforcée dans les années 1990-2000 du fait du développement du tourisme à Valparaiso. Dans ce contexte, l'inscription d'une partie du centre au Patrimoine mondial en 2003 a provoqué une série de conflits où surgissent différents discours sur l'histoire, la mémoire et le patrimoine de la ville. Les habitants veulent défendre un mode d'habiter le quartier qui serait spécifique et menacé, alors ser porteño (être de Valparaíso) devient une fierté partagée et défendue. Dans ce cadre, l'idée de "(re)faire quartier » engendre de nombreux discours nostalgiques pour une communauté de quartier "d'avant ».

Le quartier Esperanza est protégé comme « zone de conservation historique » depuis 2005. C'est un secteur résidentiel historiquement ouvrier mais où les habitants se considèrent aujourd'hui comme faisant partie de la classe moyenne, avec un standard de vie supérieur à celui des habitants des autres quartiers de Valparaíso. Il se compose en grande partie de propriétaires qui ont vécu ou qui sont héritiers de cette histoire ouvrière. Le quartier n'a pas connu de grands changements urbains comme d'autres secteurs de la ville. II n'a pas non plus connu de changements socio-économiques récents. Cependant, la nostalgie pour une communauté de quartier est omniprésente dans les narrations et les conversations quotidiennes des habitants appartenant aux 
anciennes familles. Il s'agit d'une nostalgie pour une idée de « faire quartier » et « faire communauté » qui, selon eux, serait en train de disparaître.

$C^{\prime}$ est dans ce contexte que nous avons voulu comprendre le sentiment de perte de l'identité du quartier partagé par ces anciennes familles, en particulier les adultes, héritiers des mémoires et souvenirs du quartier de leurs parents. À partir d’un travail de terrain ethnographique, nous avons pu questionner le rôle du changement générationnel dans l'activation d'une nostalgie collective pour le « quartier d'avant " (tel que les enquêtés le conçoivent aujourd'hui). Cette nostalgie se fonde avant tout sur les souvenirs d'expériences personnelles ou familiales dans le quartier qui conduisent les habitants à imaginer une communauté de quartier disparue qui se manifestait par des règles et normes de convenance partagées. Même s'ils connaissent l'histoire ouvrière de ce secteur de la ville et les mémoires sociales associées, ils ne se considèrent pas comme faisant partie de la classe ouvrière, estimant qu'ils ont accédé à une forme de mobilité sociale vers la classe moyenne et considérant qu'ils habitent dans un quartier privilégié. Par conséquent, la nostalgie ne sert pas de support à une construction identitaire collective associée à l'idée de classe ouvrière mais bien davantage à la revalorisation de la conception de ce que devrait être un quartier familial avec des règles et normes partagées par ses habitants, fondée sur une forme dépolitisée de l'idée de communauté de quartier.

Le cas du cerro Esperanza invite à penser la reconfiguration communautaire à l'échelle d'un quartier, thématique largement abordée par la sociologie britannique (Blockland, 2001, 2003 ; Evans, 2006 ; McKenzie, 2012 ; Degnen, 2016 ; Lewis, 2016), non pas du fait de changements socio-économiques ou matériels mais de changements générationnels qui conduisent à la reconfiguration des liens sociaux à l'échelle du quartier. C'est une autre catégorie de la nostalgie qui se dégage ici : l'espace du quartier a peu changé, mais le groupe social s'est transformé. Tout en servant de base à la construction d'un attachement au lieu, les présences de passés et les mémoires partagées dans le présent ne s'interprètent ni ne se vivent de la même façon selon les générations. Cette différenciation entre générations dessine une nostalgie peut-être spécifique des classes moyennes en cours d'ascension sociale. Le cas des nouvelles classes moyennes sud-africaines vient confirmer l'ambivalence des nostalgies de cette catégorie sociale.

\section{UNE CLASSE MOYENNE « DÉPLACÉE » EN AFRIQUE DU SUD : ESPACES MOUVANTS DES NOSTALGIES CITADINES}

Le sentiment nostalgique citadin n'est pas I'apanage de tel ou tel groupe citadin. II est trans-classe, trans-genre et trans-race... Un cas à cet égard intéressant est celui de la classe moyenne noire sud-africaine. Le développement rapide de ce groupe social, dit " émergent " est souvent considéré comme le principal changement des sociétés urbaines du pays. Cette classe moyenne nombreuse et très visible vit pour une part encore dans les vastes townships construits sous l'apartheid et dont certains secteurs sont aujourd'hui profondément transformés. Mais une large part de la classe moyenne noire à Johannesburg a quitté le township pour accéder à la propriété dans des complexes résidentiels nouveaux, le plus souvent périphériques, toujours sécurisés et clos.

Du township aux gated communities des suburbs, il y a donc déplacement spatial. Dans ces nouveaux paysages, les citadins retrouvent la monotonie - vastes lotissements de maisons identiques les unes aux autres, petits lots — et aussi l'éloignement — on accède 
à ces résidences en voiture seulement et elles sont souvent tout aussi loin des espaces de la centralité que l'étaient les townships. Ils trouvent aussi une relative sécurité dans ces espaces fermés et gardés dont l'accès est censé être strictement contrôlé, mais ils ont perdu les relations de voisinage qui sont constitutives souvent de la vie du township. Dès lors : nostalgie... car le déplacement spatial provoque un sentiment de déplacement culturel qui fait que l'on ne se sent pas « à sa place».

La littérature contemporaine rend compte du phénomène, avec distance et humour car ne s'agit-il pas d'une nostalgie de "little rich girl »? Le grand romancier Zake Mda, dans Black Diamond (2009), décrit la vie d'un couple de cette nouvelle classe moyenne qui a quitté Soweto et la manière dont Soweto, espace emblématique de la ségrégation et de l'oppression de l'apartheid, a pu devenir un espace de la nostalgie : "More than anything else, former sowetans return to Soweto for the homeliness as well as the nostalgias : being in Soweto is like being enveloped in the ample bosom of a much-loved matriarch. [...] It is a place of nostalgia, and nostalgia does have a healing effect. Nostalgia is analgesic - it numbs you into a world where things were better, even though no one realised it at the time» (p. 28).

Ce qu'ils regrettent? C'est à proprement parler proustien : "For the Sunday lunch of chakalaka, curried chicken, dombolo steamed bread soaked in tomato and onion gravy, fried cabbage, mashed pumpkin, green beans cooked with potatoes and grated carrots, beetroot salad, custard and jelly; and to be fussed over by aunts and uncles and neighbours ; and to talk soccer with people who really know something about the " beautiful game "; and to enjoy the latest gossip in the taverns» (p. 59).

Si les nostalgies citadines en Afrique du Sud ont longtemps porté surtout sur les quartiers détruits par l'apartheid, c'est-à-dire les anciennes locations de Sophiatown ou District Six, quartiers pré-apartheid dont les habitants ont été déplacés dans les townships, les espaces nouveaux de nostalgie sont désormais, pour certains, les townships eux-mêmes. Une nostalgie qui n'est pas facile à dire, ni même à clairement identifier car au même moment la course à la propriété dans une suburb se poursuit tant elle est vécue comme le signe de l'ascension sociale. Kopano Matlwa décrit dans Coconut (2007) les affres d'une famille noire dans un complexe résidentiel de suburb. La famille vient d'Atteridgeville, un des grands townships proche de Pretoria et elle s'est installée dans le complexe de Little Valley : "Little Valley Country Estate sells itself as "your rustic escape from the rat race". Daddy says that there were many such developments coming up in the city when he bought our house because South Africans were attracted to the idea of a residential area right in the melting pot of the country but even more so to ones that also assured the 24-hour a day maximum security mandatory for survival in Johannesburg » (p. 74). Le narrateur du roman est une petite fille, Fikile, et c'est du déplacement culturel qu'elle souffre : "Inside my home it is not the smell of sauteed prawns and ricotta stuffed pasta with mushroom sauce that wafts into the garden, but rather the sharp smell of mala le mogodu » (p. 75). Et quand la grand-mère de Fikile vient du township pour organiser les cérémonies qui vont ancrer la famille dans sa nouvelle maison, ce qui suppose d'abattre un animal dans la cour (et de boire force bières aussi), on invite toute la famille élargie... jusqu'à ce que les gardes de la compagnie privée de sécurité du quartier viennent interrompre les festivités qui contreviennent au règlement intérieur de la résidence.

Mais cette nostalgie-là ne peut être dite car il n'est pas non plus question de retour en arrière vers le township... Quand la mère de Fikile, trompée par son mari, envisage le divorce, ses parents lui disent : "Where do you have to go ? [...] return to the dreaded location? » (p. 13) II faut donc vivre là, dans la résidence si peu humaine, là où " we do not belong. [...] we are shackled [...] They will shout "stop acting black! " [...] are we not black, Father? No, not in the malls, Lord. We may not be black in restaurants, in suburbs and in schools» (p. 31). 
Les deux romans décrivent la solitude et le regret, celui de la perte des relations sociales de l'ancien environnement urbain, celui de vivre dans des quartiers où " the neighbours are the cars you see parked in their driveways and the children are the tennis balls that fly over the wall and into your pool » (Coconut, p. 89), espaces de l'ennui...

Les deux cas révèlent une relation ambiguë entre nostalgies, espaces et mobilité sociale. Dans le premier cas, les générations adultes des anciennes familles du quartier Esperanza se revendiquent de la classe moyenne chilienne, invisibilisant le passé ouvrier de ce secteur, mais en même temps regrettent le temps d'une communauté de quartier imaginée aujourd'hui comme plus homogène et soudée. Dans le second cas, les familles noires sud-africaines, tout en cherchant à montrer et confirmer leur mobilité sociale par une mobilité géographique, doivent vivre avec le regret des règles de convenance de leur quartier d'avant et avec les conflits d'usage permanent entre leurs pratiques et les règles de leur nouveau lieu de vie. Pour ces citadins venus des townships, c'est la nostalgie aussi du «bon voisinage » qui est centrale, associée aux idées de liberté et d'animation. Le complexe devient le lieu présent de l'ennui, le lieu d'une sorte d'exil intra-urbain, qui vous donne la sécurité mais vous interdit d'être vous-même, le lieu qui vous change.

Dans les deux cas, ces changements socio-spatiaux conduisent progressivement à un repli sur un espace social privatisé et limité à la famille où la nostalgie pour une communauté imaginée perdue domine. Cette privatisation de l'espace social conduit donc dans les deux cas à un désengagement social (et politique) pour le quartier, à la déstructuration du sentiment collectif d'appartenance et plus généralement pour l'espace public (au sens matériel comme politique).

\section{Conflits de nostalgies}

Les groupes sociaux sont producteurs de différentes nostalgies et la nostalgie est productrice d'une grande diversité d'espaces sociaux dans la ville. Ceux-ci ne se juxtaposent pas seulement, ils se superposent, se chevauchent ou s'emboîtent. $\mathrm{C}^{\prime}$ est dans ce contexte que les discours et les pratiques nostalgiques révèlent des conflits et désaccords d'interprétation des passés dans le présent. Les choix $d$ 'interprétation reflètent d'ailleurs des relations de pouvoir entre groupes et institutions dans un même espace. Les espaces nostalgiques sont associés à des groupes sociaux formés à partir d'histoires partagées (vécues ou imaginées) dans le présent qui sont intimement liées à leur espace de vie commun. Mais, dans un même espace coexistent différentes représentations des passés et en conséquence différentes nostalgies.

Les études de cas présentées dans les parties précédentes, que ce soit au Chili ou en Afrique du Sud, mettent en évidence la manière dont le désir nostalgique pour le «quartier d'avant » ou pour la communauté imaginée (Anderson, 1991) du quartier d'avant, prend forme socialement et matériellement en ville. Mais ils font aussi apparaittre la vigueur des frontières symboliques dans la ville. Frontières générationnelles et frontières sociales entre différents groupes : ceux qui partagent 
la même nostalgie, c'est-à-dire la même interprétation du passé, et ceux qui n'ont pas de nostalgie ou qui n'ont pas la même interprétation ; ceux qui vivent dans le regret de ce que le quartier était et ceux qui vivent dans celui du lieu qu'ils ont dû quitter. Ces coprésences sont d'autant plus fréquentes que la ville contemporaine est peut-être plus que jamais caractérisée par des distinctions sociales entre le mobile et l'immobile, l'enraciné et le déraciné, le natif et l'étranger (Comaroff et Comaroff, 2010). Le cas de l'ancienne cité de Comet (Gervais-Lambony, 2012), à l'est de Johannesburg dans l'actuelle municipalité d'Ekurhuleni, est un bel exemple du rôle des nostalgies dans l'approfondissement de ces distinctions mais aussi de la concomitance de nostalgies individuelles vraiment vécues et d'un discours nostalgique porté collectivement par un groupe pour défendre ses intérêts propres et rejeter ceux qui sont définis comme "autres ».

\section{COMET : LA NOSTALGIE DE L'INDÉFENDABLE ?}

Comet était un ensemble de logements pour salariés d'une mine d'or qui a fermé depuis longtemps. Les logements collectifs des mineurs ont été détruits, reste seulement un petit ensemble de maisons individuelles qui étaient destinées à loger les employés les plus privilégiés (techniciens, mécaniciens, infirmiers, chauffeurs...). En 2010, au moment de la première enquête de terrain, les habitants légaux de ces logements sont toujours locataires de la compagnie minière, certains travaillent encore pour elle mais sur d'autres sites, d'autres sont en retraite, beaucoup de veuves aussi continuent d'occuper ces logements. Mais le système économique et territorial qui justifiait l'existence du quartier n'existe plus. Pour accroître leurs revenus beaucoup de résidents ont loué ou sous-loué leur logement ou une partie de leur logement, et une large partie des habitants du quartier n'a aucun rapport direct avec l'activité minière et beaucoup sont des migrants étrangers originaires surtout du Mozambique et du Lesotho.

Lors des entretiens de terrain les résidents les plus anciens s'expriment sur le passé de Comet avec une profonde nostalgie. Ils décrivent le temps de la mine, quand la cité était bien entretenue, quand la vie collective s'organisait au rythme défini par la mine et sa politique paternaliste : projections de films dans le hall communautaire, distributions de cadeaux aux enfants à noël, et chaque semaine distribution de lait, de pain et de viande, relations de voisinages intenses entre familles de collègues, sécurité aussi dans ce petit lotissement dont les entrées étaient contrôlées. Mais bien sûr ils disent aussi qu'ils savaient bien être des privilégiés d'un système injuste : "We knew the system was bad, but we were never hungry, and we were helping our families outside. "

À partir de 2010, ces nostalgies individuelles ont servi de ferment à un mouvement collectif qui visait à " sauver » la cité. En effet, avait été annoncée la vente des terrains et des maisons par la compagnie minière à un promoteur privé qui prévoyait la construction sur le site d'un lotissement résidentiel. Dès lors les habitants se mobilisèrent, mais seulement les habitants légaux : il s'agissait de se défendre et de se rassembler autour d'un discours sur le passé du quartier. Des négociations s'engagent alors entre la compagnie minière, le promoteur, la municipalité d'Ekurhuleni et les résidents. Tout I'enjeu est de savoir quelle partie de la cité ne sera pas détruite et quelles compensations seront accordées aux anciens habitants.

Un beau mouvement social local de défense d'un quartier ancien contre le déploiement de la ville neo-libérale portée par les investisseurs privés? Certes, mais aussi un mouvement social conservateur et nimbyste: les étrangers, les sous-locataires, ne peuvent partager ni les sentiments nostalgiques ni les droits à rester ou à compensation. Ils sont assez rapidement victimes d'évictions brutales, ce d'autant plus quand ils sont 
de nationalités étrangères (dans un contexte où les violences contre les " étrangers » sont récurrentes dans les métropoles du pays). L'intervention des autorités municipales est aussi un tri : il s'agit de savoir qui a des droits, et qui peut le prouver.

En 2015, face aux pressions de l'association de résidents, le promoteur renonce à son projet, la compagnie minière accepte de vendre les terrains et les maisons à moindre coût à la municipalité. Celle-ci engage une procédure de cession aux habitants " légaux ». Victoire des nostalgiques d'un temps qui fut pourtant celui de l'apartheid, nostalgie pour l'indéfendable? Le résultat est aussi la discrimination des plus fragiles et de ceux dont la nostalgie n'est pas celle du passé-ici est celle d'un passé-ailleurs, lointain qu'ils ont du quitter.

Dans le cas de Comet, comme dans celui du quartier de Santiago centre décrit plus haut, s'opposent d'un côté le groupe mobilisé et ses sympathisants et de l'autre ceux qui ne partagent pas la même nostalgie. Ces derniers sont généralement des résidents étrangers ou nationaux mais nouveaux venus. Le groupe mobilisé par le discours nostalgique est fédéré par un but commun : défendre un quartier à partir de la réinterprétation qu'ils font d'un «avant». L'autre groupe est hétérogène et ne connait pas de cohésion interne : il s'agit de personnes n'ayant ni les mêmes trajectoires de vie, ni les mêmes modes de vie, ni les mêmes attentes. Progressivement, le premier groupe se constitue à partir du renforcement de normes sociales et comportementales et de la stigmatisation de ceux qui ne respectent pas ces normes. Nous assistons à une fragmentation sociale du quartier par le fait même de cette stigmatisation de l'autre. Phénomène qui, à Santiago, conduit le premier groupe à s'approprier l'espace matériel du quartier de manière éphémère (fête dans la rue, évènement culturel sur la place, conversation quotidienne le soir avec les voisins dans la rue) et de manière permanente (valorisation patrimoniale des maisons du secteur, plantation d'arbustes et de fleurs dans les plates-bandes des rues...). Les conséquences dans le cas de Comet sont bien plus brutales : éviction des sous-locataires ou occupants non légaux de logements. Dans ce sens, le désir nostalgique de « refaire quartier» tout à la fois fédère et divise, il conduit ces habitants à faire une séparation entre ce qui serait un « bon» et un « mauvais» voisin.

Si l'on revient sur le cas du quartier d'Esperanza à Valparaiso, on voit se dessiner d'autres lignes de fractures entre habitants, mais ces lignes de séparations entre micro-groupes sont bien plus fines. Les anciennes familles du quartier partagent à première vue un imaginaire commun de ce qu'était le quartier «avant». Face à l'idée d'une perte d'identité de quartier, ils donnent l'image d'une communauté unie où tous les membres des anciennes familles se connaissent. Dans leurs discours, ils font une différence claire entre un «nous » et «les autres» fondée sur l'idée nostalgique du «bon voisin» solidaire et proche des autres habitants, valeurs qu'ils n'associent pas aux nouveaux résidents du quartier. Cependant, au fil des conversations, on comprend que la délimitation entre le «nous» et « les autres» change en fonction du lieu de résidence dans le quartier. Tandis que pour certains, le «nous » correspond aux voisins de la même rue, pour d'autres il s'agit des personnes rencontrées de manière régulière dans 
leur quotidien. Chaque «nous » renvoie en réalité à un espace social particulier avec une interprétation nostalgique particulière du passé. Cette situation met en évidence l'existence de multiples espaces nostalgiques dans le même quartier. Espaces délimités différemment selon les habitants, leur lieu de résidence mais aussi leur génération, mais aussi définis socialement. En effet, les habitants « d'en haut » du cerro sont considérés comme plus pauvres que ceux «d'en bas ». Dans les discours des habitants d'en bas, il est clair que ceux d'en haut ne font pas parti de leur idéal nostalgique du quartier. À l'inverse, ceux d'en haut dévalorisent l'attitude hautaine de ceux d'en bas, considérant qu'ils ne font pas partie du «vrai » Esperanza.

\section{Conclusion}

Nous vivons dans des espaces qui sont à la fois « réels et imaginés » (Soja 2000). Cette affirmation d'Edward Soja s'applique aussi aux « lieux que nous avons connus» (Gervais-Lambony et Desbois, 2017). Et, ajoutons, lieux que nous avons connus et que nous habitons toujours : les travaux sur les nostalgies citadines nous montrent assez que les existences humaines ne se déroulent pas dans l'ici et maintenant, ni seulement dans le lieu présent mais aussi dans les lieux passés, ni dans une chronologie simple de moments successifs. Nous ne vivons donc pas dans un lieu unique, comme l'avait pressenti le mouvement simultanéiste à la suite de Blaise Cendrars : nous sommes en même temps dans les lieux de notre présent et dans ceux de notre passé, et ce plus encore dans les contextes urbains que dans la condition humaine en général. La nostalgie, quelle que soit sa forme, est la manière dont se font présents le lieu et le temps passés dans le lieu et le moment actuel, les uns et les autres réels et imaginés à la fois. Cette résurgence du passé dans l'espace et le temps présent provoque une émotion liée au souvenir, et dans cet événement les objets rencontrés dans l'espace, tel le pont Mirabeau de Guillaume Apollinaire, jouent souvent le rôle essentiel de déclencheurs, non pas en soi mais parce qu'ils évoquent une relation affective. Cette relation entre mémoire et espace était déjà tant soulignée par Maurice Halbwachs, ne serait-ce que par le fait que le dernier chapitre de son livre de 1950, largement mobilisé ici, traite de la relation citadine à l'espace. Et ceci, concerne tout autant les citadins qui n'ont pas changé de lieu (leur nostalgie porte sur ce même lieu tel qu'il a été et tel qu'ils l'imaginent avoir été) que ceux qui en ont changé (leur nostalgie porte sur le lieu qu'ils ont quitté, ou les lieux successifs qu'ils ont connus). La vigueur de ces imaginaires est si grande qu'elle sert bien souvent de fondement à des constructions identitaires, à établir des lignes de séparation entre soi et l'Autre ; mais on a vu aussi toutes les ambiguïtés du passage des imaginaires individuels aux discours porteurs de mobilisation collective qui conduisent à l'action pour construire le futur. Les recherches sur le sujet peuvent relever d'une géographie humaniste, ou de l'expérience, et se concentrer dès lors plutôt sur la dimension individuelle de la nostalgie. Mais ces dimensions 
individuelles fondent aussi des divisions sociospatiales intra-urbaines à l'échelle du quartier ou de la ville. La difficulté des analyses en termes de nostalgie est qu'elles ne peuvent donc se limiter à un seul versant de la question, que les interrelations entre nostalgies individuelles et constructions sociales collectives fonctionnent à double sens. Apparait aussi toute la complexité des interrelations entre espace matériel et espace imaginé, l'un étant producteur de l'autre et inversement. À cette relation entre nostalgies et matérialité (passée et présente), le géographe sera particulièrement sensible parce qu'elle rend son propos immédiatement pertinent sur un sujet qui pourrait sembler relever d'abord de l'anthropologie ou de la sociologie.

Dans les contextes urbains contemporains, ces différentes questions ont pris une importance toute particulière. Trois facteurs l'expliquent sans doute : des politiques de patrimonialisation "par le haut» se multiplient en même temps que les références au passé circulent à l'échelle planétaire et que les grands projets urbains sont porteurs d'imaginaires devenus globaux ; les mouvements sociaux de résistance au changement ou appelant au changement se fondent plus que jamais sur des références à des passés locaux réels et imaginés; les mobilités accrues, à toutes les échelles (de l'intra-urbain à l'international) font coexister sur les mêmes lieux des nostalgies différentes qui deviennent facilement contradictoires puis conflictuelles. Dans ce contexte, quelle nostalgie est plus légitime qu'une autre ? Quelle nostalgie n'est pas excluante de l'Autre, Autre défini comme «étranger » parce que ne partageant pas le même imaginaire du passé du lieu ? Nous avons fait le choix d'aborder ici la question " par le bas », c'est-à-dire en centrant notre interrogation sur les émotions vécues et exprimées par les citadins. Ceci soulève deux questions pour des travaux à venir, l'une d'échelle en quelque sorte, l'autre méthodologique.

Réfléchir sur les échelles auxquelles sont produites les nostalgies suppose de travailler sur les effets locaux de décisions d'aménagement urbain qui mobilisent des références nostalgiques au passé prises «par le haut». Les projets de rénovation de centres anciens tout comme les grands projets urbains de nouveaux quartiers foisonnent de références à des modèles urbains passés. Comment s'articulent-ils avec les vécus citadins ? Et les vécus de quelles catégories de citadins : nostalgies des classes populaires accaparées par les classes moyennes et supérieures dans le cas des quartiers gentrifiés ; exotismes des quartiers ethniques adaptés pour attirer le touriste étranger; mémoire de luttes sociales détournées pour servir de référent à un projet urbain néolibéral ? On peut imaginer toutes les contradictions ou toutes les complémentarités, mais la même question de méthodologie se pose à chaque fois que l'on cherche à étudier sentiments et émotions. En effet, sur nos terrains, la nostalgie est apparue comme par détour : menant des travaux de recherche géographiques sur le présent nous avons entendu parler du passé avec regret. Dans le discours des citadins, le présent nous a donc semblé se juger à l'aune du passé. À partir de là nous avons fait le choix de proposer la catégorie d'analyse de la nostalgie. Mais pour poursuivre dans cette direction il faut proposer aussi de dépasser le niveau du discours nostalgique, 
d'où les approches par les pratiques : la manière de bâtir ou décorer sa maison, la manière de cuisiner, la manière de circuler et de se mouvoir dans l'espace, la production des lieux et des modes de sociabilités, l'ensemble des " arts de faire ", enfin, doit être mobilisé. Le champ à ouvrir en géographie est donc aussi celui de méthodes de recherche adaptées à une géographie des émotions. Et certainement il faudra faire "feu de tout bois", être avec, vivre avec, idéalement éprouver avec. Il s'agira en tout cas d'écouter ensemble la même musique, de parcourir ensemble les rues de la ville... de guetter le surgissement émotions. Ceci va même au-delà de la méthode d'enquête dite "ethnographique » à laquelle nous nous sommes référés à plusieurs reprises dans les pages qui précèdent, c'est une des raisons pour lesquelles nous avons ici mobilisé dans un cas la littérature pour analyser les vécus citadins, l'étude des productions artistiques de toutes natures est certainement à ranger dans nos outils pour une géographie des nostalgies.

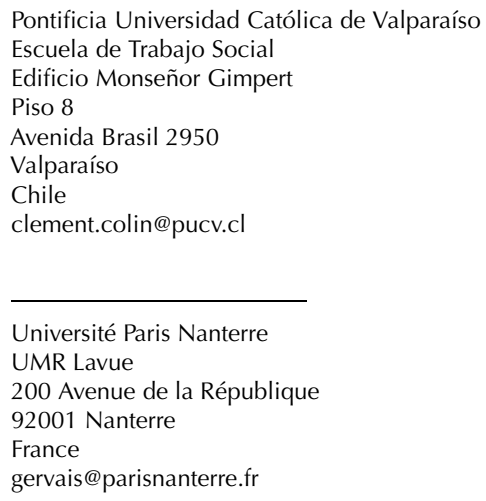

\section{Bibliographie}

Anderson, B. (1991), Imagined Communities : Reflections on the Origin and Spread of Nationalism, Londres, Verso, 224 p.

Angé O., Berliner D. (dir.) (2015), Anthropology and Nostalgia, Oxford, Berghahn, 248 p.

Akassegawa, G. (1987), Hyperart: Thomason, Tokyo, Chikuma Shobo, 416 p.

Appadurai, A. (1996), Modernity at large. Cultural dimensions of globalizations. Minneapolis, University of Minnesota Press, 229 p.

Berdahl D. (1999), «(N) Ostalgie for the present. Memory, longing, and East German things », Ethnos, 64 (2), p. 192-211.

Berliner, D. (2012), "Multiple nostalgias : The fabric of heritage in Luang Prabang », The Journal of the Royal Anthropologicallnstitute, $\mathrm{n}^{\circ} 18$, p. 769-786.

Berliner, D. (2013), « Nostalgie et patrimoine. Une esquisse de typologie », in Fabre, D. Emotions patrimoniales, Paris, Maison des sciences de l'homme, p. 393-409.

Bissell, W. (2005), «Engaging colonial nostalgia », Cultural Anthropology, n 2, vol. 20, p. 215-248. 
Blokland, T. (2001), "Bricks, mortar, memories : neighbourhood and networks in collective acts of remembering ", International Journal of Urban and Regional Research, n 25, p. 268-283.

Blokland, T. (2003), Urban Bonds, Cambridge, Polity, 256 p.

Blunt, A. (2003), "Collective memory and productive nostalgia: Anglo-Indian homemaking at McCluskieganj, Environment and Planning D : Society and Place, $\mathrm{n}^{\circ} 21$, p. 717-738

Bonnett, A., et Alexander, C. (2013), "Mobile nostalgias: Connecting visions of the urban past, present and future amongst ex-residents ». Transactions of the Institute of British Geographers, $n^{\circ} 38$, p. 391-402.

Bonnett, A. (2015), The Geography of Nostalgia : Global and Local Perspectives on Modernity and Loss, Abingdon, Taylor \& Francis, 312 p.

Boym, S. (2001), The Future of nostalgia. New York, Basic Books, 432 p.

Cassin, B. (2013), La nostalgie. Quand donc est-on chez soi ?, Paris, Autrement, 152 p.

Certeau M. de (1994 [1980]), L'Invention du quotidien, t. 2, Paris, Gallimard, 448 p.

Colin, C. (2016), "Défendre et protéger son quartier de la destruction: les émotions dans la mobilisation d'habitants contre les projets immobiliers dans le quartier Matta Sur, Santiago du Chili », Carnets de Géographes, 9. DOI : 10.4000/cdg.555. Disponible sur : http://journals. openedition.org/cdg/555.

Corbin A., Courtine J.-J, Vigarello G. (2016), Histoire des émotions, Paris, Le Seuil (3 volumes).

Crapanzano, V. (1994), "Réflexions sur une anthropologie des émotions », Terrain, n²2, p. 109-117.

Comaroff, J., et Comaroff, J. (2010), Zombies et frontières à l'ère néolibérale. Paris, Les Prairies Ordinaires, $192 \mathrm{p}$.

Desbois, H. et Gervais-Lambony P. (2017), Les lieux que nous avons connus, Nanterre, Presses Universitaires de Paris Nanterre, $144 \mathrm{p}$.

Didier, S. (2018), « Droit de mémoire, droit à la ville », Géographies et cultures, n 105, p. 135-152.

Edwards, J. (2000), Born and Bred, Oxford, Oxford University Press, 280 p.

Evans, G. (2012), «The Arboriginal People of England : the culture of class politics in contemporany Britain », Focaal, 62, pp. 17-29.

Gervais-Lambony, P. (2003), Territoires citadins, Paris, Belin, 268 p.

Gervais-Lambony, P. (2012), " Nostalgies citadines en Afrique du Sud », EspaceTemps.net, (https://www. espacestemps.net/articles/nostalgies-citadines-en-afrique-sud/).

Gervais-Lambony, P. (2017), «Le tomason : un concept pour penser autrement les discontinuités et discontiguités de nos vies citadines ? », Espaces et sociétés, n 168-169, pp. 205-218.

Guinard, P. et Tratnjek B. (éd.) (2016), Géographie des émotions, Carnets de Géographes (https://journals.openedition.org/cdg/480).

Halbwachs M. (1950), La mémoire collective, Paris, PUF, 294 p.

Harvey, D. (1990), The Condition of Postmodernity, Oxford, Blackwell, 388 p.

Huyssen, A. (2003), Present pasts, urban palimpsest and the politics of memory. Stanford, Stanford University Press, $192 \mathrm{p}$.

Kitson, J., y McHugh K. (2015), "Historic enchantments - materializing nostalgia », Cultural Geographies, nº 3, vol. 22, p. 487-508.

Lewis, C. (2016), "Regenerating community? Urban change and narrative of the past ", The Sociological Review, ${ }^{\circ} 4$, vol. 64, p. 912-928. Lowenthal, D. (1985). The past is a foreign country, Cambridge, Cambridge University Press, 660 p.

Lavabre, M-C. (2012), "Circulation, internationalization, globalization of the question of memory ", Journal of Historical Sociology, juin 2012, p. 177-190.

Lowenthal, D. (2015), The past is a Foreign Country, Cambridge University Press, 402 p. 
Massey, D. (1995), « Places and their pasts », History Workshop Journal, n 39, p. 182-192.

Matlwa, K. (2007), Coconut, Johannesbourg, Jacana Media, 224 p.

McKenzie, L. (2012), "A narrative from the inside, studying St Anns in Nottingham : belonging, continuity and change », The Sociological Review, 60 (3), pp. 457-475.

Pickering M. et Keightley E. (2006), « The modalities of nostalgia », Current Sociology, n 6, vol. 54, p. 919-941.

Poulet G. (1963), L'espace Proustien, Paris, Gallimard, 224 p.

Soja E. (2000), Postmetropolis. Critical Studies of cities and regions, Oxford, Blackwell, 440 p.

Todorov, T. (2004), Les abus de la mémoire, Paris, Arléa, 60 p.

Tuan, Y-F. (1977), Space and Place. The perspective of experience, Minneapolis, University of Minnesota Press, 248 p.

Veschambre, V. (2008), Traces et mémoires urbaines. Enjeux sociaux de la patrimonialisation et de la démolition, Rennes, Presses universitaires de Rennes, 315 p.

Wheeler, R. (2016), "Local history as productive nostalgia ? Change, continuity and sense of place in rural England», Social and Cultural Geography, n 4, vol. 18, p. 1-21. 